\title{
Partial photoneutron cross section measurements on ${ }^{209} \mathrm{Bi}$
}

\author{
Ioana Gheorghe ${ }^{1,2, a}$, Dan Filipescu ${ }^{1}$, Seitaro Katayama ${ }^{3}$, Hiroaki Utsunomiya ${ }^{3}$, Sergey Belyshev ${ }^{4}$, Konstantin Stopani ${ }^{5}$, \\ Vladimir Varlamov ${ }^{5}$, Tatsushi Shima ${ }^{6}$, Yiu-Wing Lui ${ }^{7}$, Sho Amano ${ }^{8}$, and Shuji Miyamoto ${ }^{8}$ \\ 1 ELI-NP, "Horia Hulubei” National Institute for Physics and Nuclear Engineering (IFIN-HH), 30 Reactorului, \\ 077125 Bucharest-Magurele, Romania \\ 2 University of Bucharest, Post Office Box MG-11, 077125 Bucharest-Magurele, Romania \\ 3 Department of Physics, Konan University, Okamoto 8-9-1, Kobe 659-8501, Japan \\ 4 Lomonosov Moscow State University, Department of Physics, Moscow 119991, Russia \\ 5 Lomonosov Moscow State University, Skobeltsyn Institute of Nuclear Physics, Moscow 119991, Russia \\ ${ }^{6}$ Research Center for Nuclear Physics, Osaka University, Suita, Osaka 567-0047, Japan \\ 7 Cyclotron Institute, Texas A\&M University, College Station, Texas 77843, USA \\ 8 Laboratory of Advanced Science and Technology for Industry, University of Hyogo, 3-1-2 Kouto, Kamigori, Ako-gun, \\ Hyogo 678-1205, Japan
}

\begin{abstract}
New data of total and partial $(\gamma, \mathrm{xn})$ cross sections with $\mathrm{x}=1-3$ are proposed to be measured by direct neutron multiplicity sorting using Laser Compton scattering (LCS) gamma ray beams at the ELINP to solve long standing discrepancies between existing Lawrence Livermore National Laboratory (USA) and France Centre d'Etudes Nucleaires de Saclay photonuclear data. Pioneering experiments are currently performed at the NewSUBARU facility. We present here preliminary results on ${ }^{209} \mathrm{Bi}(\mathrm{g}, \mathrm{xn})$ cross section measurements.
\end{abstract}

\section{Introduction}

The Extreme Light Infrastructure - Nuclear Physics (ELINP) [1] is a large scale facility dedicated to nuclear physics studies with extreme electromagnetic fields. Currently under implementation, it is forseen to be open for public use in 2018, when it will host two high power $10 \mathrm{PW}$ lasers and a very brilliant gamma-ray beam source ( $20 \mathrm{MeV}$ maximum photon energy, up to $0.5 \%$ relative energy resolution, $\sim 10^{8}$ photons per second flux within the bandwidth [2]). The experimental conditions given by the unique properties of the future ELI-NP $\gamma$-ray beams will allow to perform precise measurements of photonuclear reactions cross sections in the giant dipole resonance (GDR) and Pygmy dipole resonance (PDR) energy range.

Partial $(\gamma, \mathrm{xn})$, with $\mathrm{x}>1$ and total photoneutron reaction cross section measurements represent an important subject within the ELI-NP physics program [3]. The majority of photoneutron reactions were investigated using quasi-monoenergetic positron in flight annihilation photon beams at the Lawrence Livermore National Laboratory (USA) and France Centre d'Etudes Nucleaires de Saclay. There are significant discrepancies between the cross sections measured at the two facilities that can not be resolved in a systematic manner [4,5]. To solve this long standing problem, a direct neutron multiplicity (DNM) sorting technique of obtaining $(\gamma, \mathrm{xn})$ cross sections, where $\mathrm{x}=1-4$, has been developed [6] and a new campaign of such measurements is proposed to be performed using the ELI-NP gamma beam system.

$\overline{\text { a e-mail: ioana.gheorghe@eli-np.ro }}$
The neutron sorting method relies on the constant neutron detection efficiency relative to the energy of the reaction neutrons. For this, we have recently developed a flat efficiency neutron detector composed of ${ }^{3} \mathrm{He}$ counters embedded in a moderator. The method has been applied for the first time to ${ }^{209} \mathrm{Bi}$ at the experimental hutch GACKO (Gamma Collaboration Hutch of Konan University) of the $\gamma$-ray beam line of the NewSUBARU synchrotron radiation facility [7]. Photoneutron ${ }^{209} \operatorname{Bi}(\gamma, \mathrm{xn})$ with $\mathrm{x}=1-4$ reaction cross section measurements have been performed by using laser Compton scattered $\gamma$-ray beams with energies up to $42.5 \mathrm{MeV}$.

This is the first of a series of experiments dedicated to partial photoneutron cross section measurements for the IAEA-CRP F41032 which make use of the new neutron multiplicity sorting method. We report here on the experimental technique, data analysis and preliminary results.

\section{Experimental procedure}

LCS $\gamma$-ray beams were produced with a high power Q-switch Nd: $\mathrm{YVO}_{4}$ laser INAZUMA (Spectra-Physics) and relativistic electron beams. The $(\gamma, n)$ reaction was investigated starting from the vicinity of the neutron emission threshold $\left(S_{n}=7.46 \mathrm{MeV}\right)$ up to the double neutron emission threshold $\left(\mathrm{S}_{2 n}=14.35 \mathrm{MeV}\right)$ using electron beams of 610 to $910 \mathrm{MeV}$ energy and the laser operated in the first harmonic $(\lambda=1064 \mathrm{~nm}$; power $=40 \mathrm{~W})$. The $(\gamma, 2-4 \mathrm{n})$ reactions were investigated from 14.35 up to $42 \mathrm{MeV}\left(\mathrm{S}_{3 n}=22.45 \mathrm{MeV}, \mathrm{S}_{4 n}=\right.$ $29.40 \mathrm{MeV}, \mathrm{S}_{5 n}=37.97 \mathrm{MeV}$ ) using electron beams of

(C) The Authors, published by EDP Sciences. This is an Open Access article distributed under the terms of the Creative Commons Attribution License 4.0 (http://creativecommons.org/licenses/by/4.0/). 


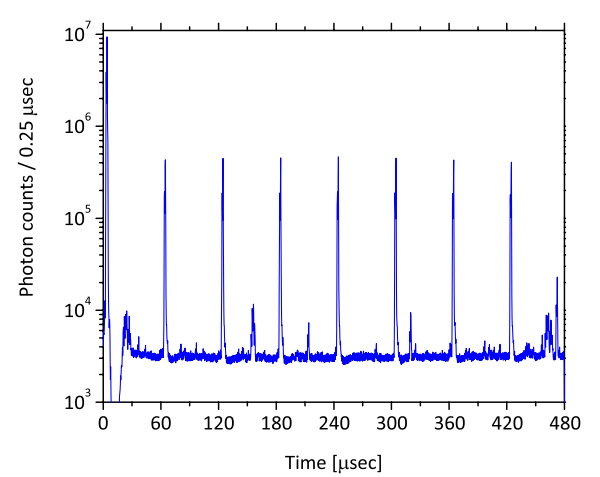

Figure 1. Incident LCS $\gamma$-ray beam time spectra recorded with the $\mathrm{NaI}(\mathrm{Tl})$ detector. The time is relative to the clock signal.

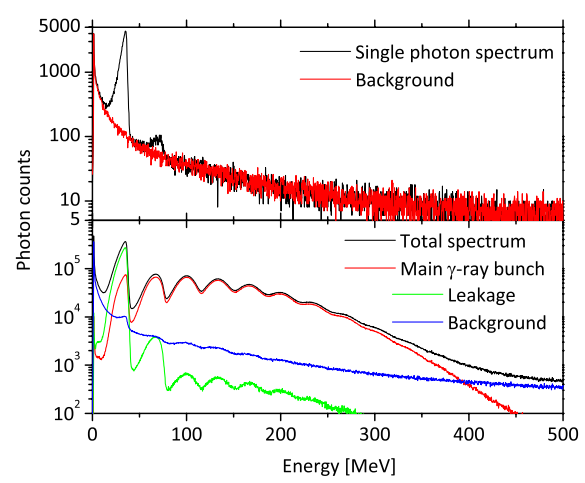

Figure 2. $\mathrm{NaI}(\mathrm{Tl})$ detector response to $33.3 \mathrm{MeV}$ maximum energy LCS $\gamma$-ray beams. Top figure: the single photon (black) and background (red) spectra. Bottom figure: the total $\gamma$-ray spectrum (black) along with its main (red), leakage (green) and background (blue) components.

640 to $1108 \mathrm{MeV}$ energy and the laser operated in the second harmonic $(\lambda=532 \mathrm{~nm}$; power $=14 \mathrm{~W})$.

The DNM sorting technique requires that a $\gamma$-ray bunch impinged on the target can generate maximum one nuclear reaction and the emitted reaction neutrons have enough time to moderate and be recorded by the detector before the arrival of the following $\gamma$-ray bunch. The reaction rate limitation is given by an appropriate combination of incident $\gamma$-ray flux, target thickness and reaction cross section. These first two quantities have been chosen for each energy point using cross section estimations provided by statistical model calculations and previous experimental data.

Based on Geant 4 simulations, the moderation time chosen for this experimental campaign was of $480 \mu \mathrm{s}$ between consecutive $\gamma$-ray bunches. Because the maximum time interval between the INAZUMA laser photon pulses is of $60 \mu \mathrm{s}$, an additional optical system was used to obtain the desired beam time structure. A Pockels cell and a polarizer were used to block 7 out of 8 consecutive laser pulses. The working parameters of the optical system were chosen as to maximize both the transmission for allowed laser components and the stopping power for the blocked laser components. Nevertheless, the laser transmission minimum for the blocked components was not zero, as can be observed from time distribution of the laser beam, $\gamma$-ray beam and neutron moderation.

The $\gamma$-ray beam flux was monitored during each neutron measurement run with a 8 in. $\times 12$ in. $\mathrm{NaI}(\mathrm{Tl})$ detector placed at the end of the LCS $\gamma$-ray beam line.

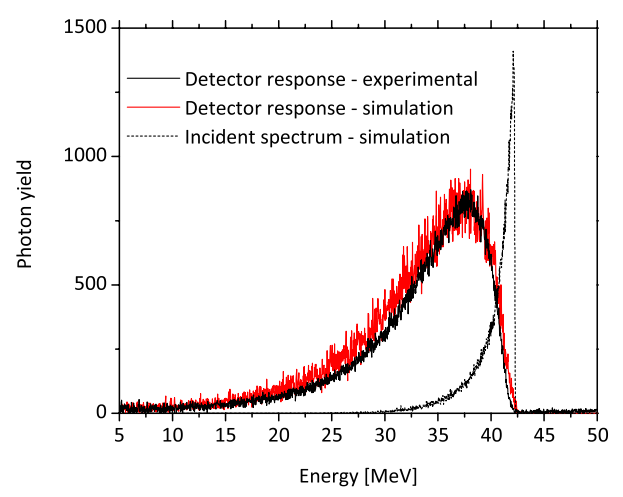

Figure 3. Experimental (solid black) and simulated (red) $\mathrm{LaBr}_{3}:$ Ce detector response to $42.5 \mathrm{MeV}$ maximum energy LCS $\gamma$-ray beam, and the simulated incident $\gamma$-ray beam (dotted black). The $\gamma$-rays were generated using a $1108.4 \mathrm{MeV}$ electron beam and the laser operated in the second harmonic.

Figure 1 shows a $33.3 \mathrm{MeV}$ maximum energy incident LCS $\gamma$-ray beam time spectra, where the time is relative to the previous clock signal.

The high amplitude time peak in the vicinity of $0 \mu \mathrm{s}$ represents the main LCS $\gamma$-ray bunches, corresponding to the allowed laser pulses. The lower amplitude time peaks shifted between them by $60 \mu$ s represent the leakage LCS $\gamma$-ray bunches, corresponding to the partially blocked laser pulses. The total energy spectrum of the $\gamma$-rays recorded during the neutron measurement is displayed in the bottom Fig. 2 (black) along with its three components identified above: the main LCS bunches $\gamma$-rays (red), the leakage (green), and the background (blue), which are discriminated by applying time gate conditions.

Because the duration of the electron beam bunch is much smaller $(2 \mathrm{~ns})$ than the laser one $(60 \mathrm{~ns})$ and the laser - electron Compton scattering cross section is small, the LCS $\gamma$-rays are generated in bunches corresponding to each laser light pulse following a Poisson probability distribution. The mean value of the Poisson distribution mainly depends on the laser power and electron beam current.

Therefore, the main LCS $\gamma$-ray spectrum displays a Poisson distributed pile-up structure with a high photon multiplicity average of 3.84 photons per bunch, while the partially blocked leakage component is characterized by a lower average of 1.08 photons per bunch. The number of recorded LCS $\gamma$-ray photons was obtained with the pile up method described in [8], where the pile-up spectra are deconvoluted using single-photon spectra. The single photon spectra are recorded before or after each neutron measurement run by operating the laser at reduced power, where it is most likely to measure only one photon at a time. The $33.3 \mathrm{MeV}$ maximum energy LCS $\gamma$-ray beam single-photon spectrum is displayed in the top Fig. 2.

The $\gamma$-ray beam energy profile was measured with a large volume $3.5 \mathrm{in} . \times 4$ in. lanthanum bromide $\left(\mathrm{LaBr}_{3}: \mathrm{Ce}\right)$ detector as described in [9]. Figure 3 shows the experimental detector response (solid black line) to $42.5 \mathrm{MeV}$ maximum energy LCS $\gamma$-rays along with GEANT4 [11] simulations of the detector response function (solid red line) and the $2.5 \%$ energy spread in full width at half maximum incident $\gamma$-ray beam (dashed 


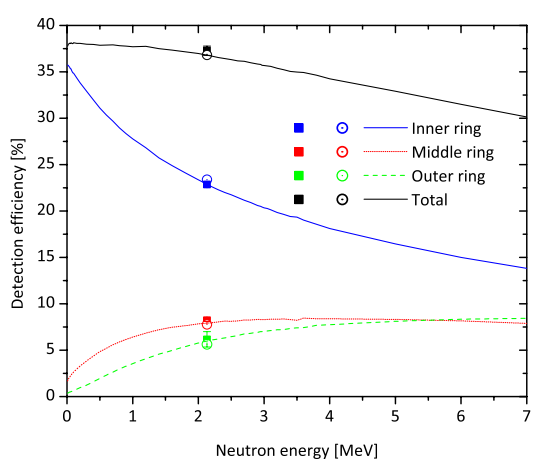

Figure 4. The detection efficiencies of the inner (blue), middle (red), and outer ring (blue) and the total efficiency (black) for s-wave neutrons obtained by: MCNP simulations using neutron evaporation spectra (curves), MCNP simulation of a ${ }^{252} \mathrm{Cf}$ source (open dots) and by experimental measurements with a calibrated ${ }^{252} \mathrm{Cf}$ (full squares).

black line). The experimental response function is well reproduced by the GEANT4 simulation.

A flat efficiency neutron detection system based on the slowing down of neutrons followed by their conversion into charged particles through the ${ }^{3} \mathrm{He}(\mathrm{n}, \mathrm{p}) \mathrm{t}$ reaction has been recently developed within the ELI-NP Konan University collaboration. Such type of detector is mandatory for the DNM sorting technique applied to obtain absolute $(\mathrm{g}, \mathrm{xn})$ reaction cross sections, as described in length in [6].

The flat efficiency detector (FED) consists of three rings of 4, 9 and $18{ }^{3} \mathrm{He}$ counters (10 atm) located at $5.5 \mathrm{~cm}, 13 \mathrm{~cm}$ and $18 \mathrm{~cm}$ from the $\gamma$-ray beam axis, respectively. Neutron detection efficiency simulations have been performed using the MCNP [10] simulation package considering s-wave evaporation neutron sources placed in the center of the moderator. The evaporation spectra were obtained by applying the Weisskopf-Ewing model, where the spectrum is given by:

$$
p(E)=\frac{E}{\theta^{2}} \exp (-E / \theta),
$$

where $E$ is the neutron energy and $\theta$ is the residual nuclear temperature of the nucleus which emitted the neutron and the average energy of the neutron emission spectrum $E_{\mathrm{avg}}^{\mathrm{ev}}=2 \theta$.

The detection efficiency was measured to be $37.27 \pm$ $0.82 \%$ at $2.13 \mathrm{MeV}$, the average energy of the ${ }^{252} \mathrm{Cf}$ neutron spectrum. The measurement was performed with a ${ }^{252} \mathrm{Cf}$ source whose absolute emission rate of (1.62 \pm $0.04) \times 10^{4}$ neutrons per second was calibrated at the National Metrology Institute of Japan in May 2015. As shown in Fig. 4, the experimental calibration is well reproduced by the MCNP simulations performed considering Weisskopf-Ewing evaporation spectra and also $1.42 \mathrm{MeV}$ temperature Maxwell fission spectra. All simulation results are displayed for the average energy of the neutron spectra. The total efficiency is $38.0-35.7 \%$ over a neutron energy range $0-3 \mathrm{MeV}$ and $38.0-32.9 \%$ over $0-5 \mathrm{MeV}$.

\section{Data analysis}

We give here details on the preliminary data analysis of neutron moderation time spectra, from which we

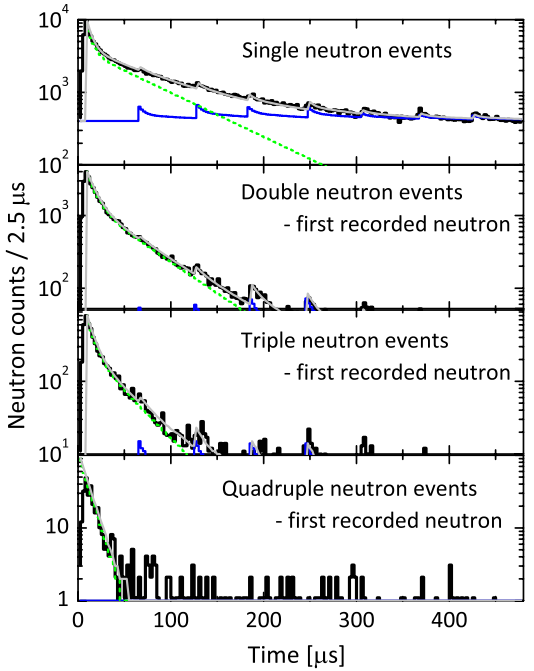

Figure 5. Neutron moderation time spectra of single, double, triple and quadruple neutron events generated in ${ }^{209} \operatorname{Bi}(\gamma, \mathrm{xn})$ reactions induced by $33.3 \mathrm{MeV}$ maximum energy LCS $\gamma$-rays. The experimental time spectra (black) are displayed along with the corresponding best fit (grey). The fit functions for the main $\gamma$ ray bunches reaction neutrons (dashed green) and the background (blue) contributions are displayed separately.

determined the number of events in which only one single $\mathrm{N}_{s}$, only two - double $\mathrm{N}_{d}$, only three - triple $\mathrm{N}_{t}$ and only four - quadruple neutron events $\mathrm{N}_{q}$, were recorded by the FED. These quantities are neccesary for computing the number of $(\mathrm{g}, \mathrm{xn})$ emitted reactions $\mathrm{N}_{x}$, with $\mathrm{x}=1-4$, using the DNM sorting technique [6]. Here, by event we define the time interval between two consecutive $\gamma$-ray bunches incident on the target.

Figure 5 shows examples of neutron time moderation spectra for single, double, triple and quadruple events, respectively. For all the cases, the spectra were constructed for the first arriving neutron out of one, two, three, respectively four neutrons. In the single neutron events spectrum can be observed eight neutron moderation time curves shifted by $60 \mu$ s from one another. These structures present a very sharp rise time followed by an exponential curve. The first of such structures, starting in the vicinity of the time axis and having the highest amplitude, correponds to the reactions induced by the main $\gamma$-ray bunch. The following seven such structures, having lower amplitudes than the first, correpond to reactions induced by the leakage $\gamma$-ray bunches.

Each $\gamma$-ray beam reaction neutron time structure was fitted using a sum of two exponential functions:

$$
h_{x}(t)=A_{1 x} \cdot \exp \left(\frac{x T-t}{\tau_{1 x}}\right)+A_{2 x} \cdot \exp \left(\frac{x T-t}{\tau_{2 x}}\right),
$$

where $x$ is the index of the structure ( 0 for the main $\gamma$-ray bunch and from 1 to 7 for the succesive leakage components), and $T$ is the $60 \mu$ s interval between consecutive bunches. The entire experimental spectrum is therefore fitted using the following function:

$$
h(t)=B+\sum_{x=0}^{x=7} h_{x}(t),
$$

where $B$ is a constant background component. 


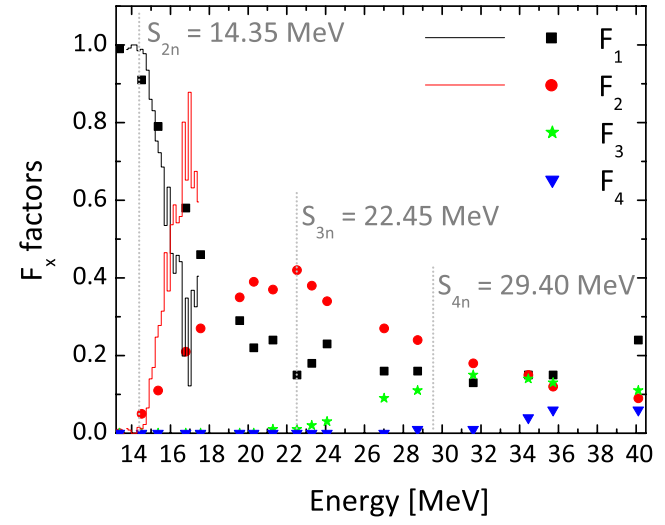

Figure 6. Experimental $F$ functions for ${ }^{209} \mathrm{Bi}$. The present results (symbols) are compared with existing Livermore data (continuous black and red lines) [12].

The number of neutrons, $N$, generated in reactions induced by the main $\gamma$-ray bunches, for each measurement point and for each type of events - single, double, triple and quadruple, was computed by subtracting the background and leakage components from the total number of neutrons:

$$
N=N_{\exp }-\int B d t-\sum_{x=1}^{x=7} \int h_{x}(t) d t
$$

\section{Preliminary results}

The neutron multiplicity sorting technique described in [6] was applied to the experimental data to obtain the number of $(\mathrm{g}, \mathrm{xn})$ induced reactions $\mathrm{N}_{\mathrm{x}}$, with $\mathrm{x}=1-4$. The results are described using the $F$ function formalism. The $F_{\mathrm{x}}$ factors are defined as:

$$
F_{\mathrm{x}}=N_{\mathrm{x}} / \sum_{i=1}^{m} \mathrm{x} \cdot N_{\mathrm{x}},
$$

where $m$ is the highest possible neutron multiplicity for a given incident energy and target nucleus, and $N_{\mathrm{x}}$ is the number of triggered $(\gamma, \mathrm{xn})$ reactions. Using this formalism, the highest possible values for $F_{1}, F_{2}, F_{3}$, and $F_{4}$ are $1,0.5,0.33$, and 0.25 , respectively. The preliminary results are displayed in Fig. 6, where for each measurement point and each reaction channel the corresponding $F_{\mathrm{x}}$ factor is represented. As can be observed, the experimental results are below the maximum allowed values for each channel. The experimental $F_{1}$ factor maintains high values even for energies above the double neutron emission threshold. A confirmation of this behavior of the F1 value is underway to investigate whether it is experimental like background neutrons produced by bremsstrahlung or physical like non-statistical effect at high energies.

\section{Conclusions}

A new direct neutron multiplicity sorting method which employs a constant neutron detection efficiency system has been applied for the first time to ${ }^{209} \mathrm{Bi}$. Photoneutron reactions have been investigated inside the $7.5 \mathrm{MeV}-42.5 \mathrm{MeV}$ energy range.

By analysing the neutron moderation time spectra and by applying the DNM sorting technique, the number of induced $(\mathrm{g}, \mathrm{xn})$ with $\mathrm{x}=1-4$ reactions were obtained. The results are within the limits imposed by the $F$ function formalism, but present a high contribution of low neutron emission multiplicity channels at energies above the double and triple neutron emission thresholds, which will be further investigated.

I.G. and D.F. acknowledge the support from the Extreme Light Infrastructure Nuclear Physics (ELI-NP) Phase II, a project co-financed by the Romanian Government and the European Union through the European Regional Development Fund - the Competitiveness Operational Programme (1/07.07.2016, COP, ID 1334). This work was supported by the IAEA under contracts 20476 and 20501 for the CRP on Updating the Photonuclear Data Library.

\section{References}

[1] www.eli-np.ro

[2] O. Adriani et al., arXiv:1407.3669 (2014)

[3] D. Filipescu et al., Eur. Phys. J. A 51, 185 (2015)

[4] V.V. Varlamov et al., Eur. Phys. J. A 50, 114 (2014)

[5] V.V. Varlamov et al., INDC(CCP) 433 (2002)

[6] H. Utsunomiya et al., I480 in this conference

[7] S. Amano et al., Nucl. Inst. Meth. A 602, 337 (2009)

[8] T. Kondo et al., Nucl. Inst. Meth. A 659, 462 (2011)

[9] D. Filipescu et al., Phys. Rev. C 90, 064616 (2014)

[10] J.F. Briesmeister, computer code MCNP, Version 4C (Los Alamos National Laboratory, Los Alamos, 2000)

[11] J. Allison et al., IEEE Trans. Nucl. Sci. 53, 270 (2006)

[12] R.R. Harvey et al., Physical Review 136(1B), 126 (1964) 